\title{
DIVERSIDAD Y COMPOSICIÓN FLORÍSTICA DE TRES TIPOS DE BOSQUE EN LA ESTACIÓN BIOLÓGICA CAPARÚ, VAUPÉS
}

\author{
Floristic composition and diversity of three forest types at Caparú, Vaupés \\ Biological Station
}

Palabras clave: Amazonía colombiana, composición florística, riqueza de especies, Terra Firme, Colina, Terraza, Igapó, Caparú, Vaupés.

Keywords: Colombian Amazon, floristic composition, Igapó, species richness, Terra Firme, forests, Colina, Terraza, Caparú, Vaupés.

\section{RESUMEN}

Los bosques amazónicos han sido incluidos entre los más diversos del planeta. Aunque en algunos países de la Amazonía existe un amplio registro de sus plantas, en Colombia hace falta un mayor esfuerzo para tener una buena idea de su diversidad y composición florística. Se levantaron tres parcelas permanentes de vegetación (DAP $\geq 10 \mathrm{~cm}$ ) de una ha en el Vaupés colombiano, en tres tipos de bosque: Colina, Terraza e Igapó (los dos primeros de Terra Firme y el tercero inundable). El objetivo fue cuantificar la diversidad y describir la composición florística en términos de índices de importancia para familias y especies. La parcela más diversa fue la de Colina ( $\alpha$ de Fisher $=160.3)$, seguida por la de Terraza $(\alpha=78.4)$ y por la de Igapó $(\alpha=$ 44.7). La familia Fabaceae s.l. fue la más importante en las tres ha, pero en el bosque de terraza la importancia de Arecaceae fue similar a la de Fabaceae. También en composición florística los tres tipos de bosque resultaron considerablemente distintos, siendo el inundable el más disímil de los tres. Sin embargo, también encontramos diferencias importantes entre los dos bosques de tierra firme, que pueden deberse a características abióticas (i.e. suelos diferentes). La alta diversidad reportada en este estudio coincide con la hipótesis que indica que las zonas más diversas de la Amazonía son la occidental y la central.

\section{ABSTRACT}

The Amazon forests are among the most diverse ecosystems in the planet. Although some countries of the Amazon Basin have a fairly complete record of their plants, in Colombia much effort is still needed to have a good idea of the plant diversity patterns and floristic composition. Three one-hectare vegetation-land plots ( $\mathrm{DBH} \geq 10 \mathrm{~cm}$ ) were established in Vaupés, Colombia, regarding three types of forest: Colina, Terraza (Terra Firme forests) and Igapó (periodically flooded). Plant diversity was estimated and floristic composition was described classifying the most important families and species. The highest diversity was found in Colina (Fisher's $\alpha=160.3$ ), followed by Terraza $(\alpha=78.4)$ and Igapó $(\alpha=44.7)$. Fabaceae s.l. was the most important family of all plots, but in Terraza forest the importance of Arecaceae was similar to that of Fabaceae. Floristic composition was also remarkably different amongst the three forest types, especially Igapó, which was the most dissimilar. However, both Terra Firme forests showed strong floristic differences, which may be caused by differences in abiotic factors (i.e. soils). High

1 Laboratorio de Ecología de Bosques Tropicales y Primatología, Universidad de los Andes, Carrera 1 No.18A-12, Bogotá. princessgrethel@gmail.com

2 Laboratorio de Ecología de Bosques Tropicales y Primatología, Universidad de los Andes, Carrera 1 No.18 -12, Bogotá. Correspondencia: pstevens@uniandes.edu.co 
plant diversity reported in this study agrees with the hypothesis that central and western Amazon forests are the most diverse of the Amazon Basin.

\section{INTRODUCCIÓN}

Los bosques amazónicos han sido incluidos dentro de los más biodiversos del planeta (Gentry 1988, 1992). Muchos estudios se han enfocado en intentar cuantificar la diversidad y describir la composición florística de la Amazonía para explicar por qué es una de las zonas terrestres más diversas del mundo (Gentry 1988, 1992; Wills et al. 1997, Terborgh \& Andresen 1998, Ter Steege et al. 2000, Colinvaux \& De Oliveira 2001, Pitman et al. 2002, Wright 2002, Jaramillo et al. 2006). Para entender los patrones ecológicos de la Amazonía a una escala continental, es importante hacer estudios a escalas locales y regionales en los que se describan y se cuantifiquen las características de los bosques amazónicos (Stropp et al. 2009).

En países como Ecuador, Perú y Brasil se han realizado muchos de estos estudios florísticos (De Oliveira \& Nelson 2001, Nebel et al. 2001, 2002; Pitman et al. 2002, Burnham 2004, Parolin et al. 2004a, Poulsen et al. 2006, Wittmann et al. 2006), por lo que se tiene una idea de la composición florística y la diversidad de la Amazonía de esos países. En Colombia, la región de la Amazonía abarca el $42.42 \%$ del territorio (483.119 $\mathrm{km}^{2}$ sensu SINCHI 2008), conforma la mayor cobertura vegetal natural del país (Carvajal et al. 1979) e incluye la totalidad de los departamentos de Guainía, Vaupés, Guaviare y Amazonas, así como gran parte de Caquetá y Putumayo (Diazgranados 1979). Aunque se han llevado a cabo muchos estudios florísticos y ecológicos, las características biológicas de la Amazonía colombiana siguen siendo muy poco conocidas en comparación con las de otros países de la cuenca (Cárdenas et al. 1997).

La diversidad y la composición florística son atributos de las comunidades que permiten su comprensión y comparación. El concepto de diversidad tiene dos componentes principales: la riqueza de especies (McIntosh 1967) y la equitatividad (Lloyd \& Ghelardi 1964). El primero se refiere al número de especies en una comunidad y el segundo a las proporciones relativas de cada especie, teniendo en cuenta que puede haber especies dominantes y especies raras en una comunidad (Krebs 1999). Por su parte, la composición florística se entiende como la enumeración de las especies de plantas presentes en un lugar, usualmente teniendo en cuenta su densidad, su distribución y su biomasa.

Los procesos que determinan la diversidad y la composición florística de los bosques son poco conocidos. Se han hecho esfuerzos, tanto a nivel global como a escalas regionales y locales, para entenderlos y describirlos. Gentry (1988) encontró que a gran escala hay una fuerte relación positiva entre la diversidad y la precipitación anual y que por el contrario la riqueza de los suelos no es un factor muy influyente en la diversidad de un lugar. Sin embargo, para él no era claro qué procesos determinan la composición florística y los patrones de dominancia a escalas locales. Se ha propuesto que factores abióticos como la riqueza y el drenaje del suelo (Jones et al. 2006) y las condiciones de un bosque regulan el número y el tipo de especies que pueden sobrevivir en él (Duivenvoorden 1996, Parolin et al. 2004b). Así, cuando las condiciones de un hábitat son hostiles, sólo algunas especies adaptadas lograrán establecerse (Duivenvoorden 1996, Parolin et al. 2004b).

La selva húmeda amazónica no puede entenderse como un enorme ensamblaje homogéneo de especies de plantas. Por el contrario, la Amazonía está compuesta por diferentes tipos de bosque, que pueden distinguirse de una forma directa describiendo las especies que los componen o de una forma indirecta por su topografía, clima o hidrología (Terborgh \& Andresen 1998). Toumisto et al. (1995), por medio del análisis de imágenes satelitales, propusieron que tan sólo en la Amazonía peruana se pueden distinguir cientos de tipos de cobertura vegetal. Sin embargo, la división del paisaje amazónico en diferentes tipos de bosque todavía es precaria (Terborgh \& Andresen 1998). Una división principal del paisaje es la de los bosques no inundables y los inundables (Terborgh \& Andresen 1998) y también son claras las diferencias florís- 
ticas al comparar lugares de suelos arcillosos con lugares de arenas blancas (Fine et al. 2004).

En la Estación Biológica Caparú, situada en el Vaupés colombiano, en la cuenca del río Apaporis, han sido descritos cinco tipos de bosque distintos, de los cuales dos son planos de inundación y tres son bosque de Terra Firme (Defler \& Defler 1996). Aunque existe una idea de la diversidad y la composición florística de éstos, no se había hecho una estimación formal de esas dos variables. Este estudio pretende llenar ese vacío, midiendo la diversidad y describiendo la composición florística de tres de los cinco tipos de bosque, estableciendo en cada uno de ellos una parcela permanente de vegetación de una hectárea e incluyendo todas las plantas de $10 \mathrm{~cm}$ o más de DAP (diámetro a la altura del pecho). Con el establecimiento de dichas parcelas de vegetación es posible comparar localmente los bosques del área de estudio y relacionarlos, a una escala mayor, con otros bosques de la Amazonía.

La Estación Biológica Caparú es y ha sido la fuente de muchos estudios científicos (Palacios-Acevedo \& Rodríguez-Rodríguez 1995, Defler 1996, 1999; Defler \& Defler 1996, Palacios et al. 1997, ClavijoR. 2005, González 2006, 2007; Vargas 2008). Los resultados presentados aquí son un complemento para esos estudios y serán una referencia para futuros estudios. Además, dado que los bosques de la Estación Biológica Caparú han sido muy poco intervenidos (Palacios \& Peres 2005), los resultados de este trabajo permitirán evaluar la importancia de los bosques del sector para establecer planes de manejo para una utilización sostenible del medio ambiente y serán un aporte al conocimiento que se tiene de la diversidad y la composición florística de la Amazonía colombiana.

\section{METODOLOGÍA}

\section{ÁREA DE ESTUDIO}

\section{Situación geográfica}

La Estación Biológica Caparú está situada en el Vaupés colombiano, en el municipio de Taraira, cerca de la frontera de Colombia con Brasil $\left(1^{\circ} 4^{\prime}\right.$ $31.6 " \mathrm{~S}, 69^{\circ} 30^{\prime} 49.5$ " W). Se encuentra a orillas del lago Taraira, a aproximadamente $100 \mathrm{msnm}$ y está comprendida por el resguardo indígena Yaigojé-Apaporis (Defler 1999). Esta estación hace parte de la cuenca del río Apaporis, catalogado como un río de aguas negras (Rangel \& Luengas 1997) y de segundo orden dentro de la cuenca amazónica (Bennet-Defler 1994, Defler \& Defler 1996). El lago Taraira es un antiguo meandro del río Apaporis, tiene $24 \mathrm{~km}$ de longitud (Defler 1991) y la fluctuación en el nivel de sus aguas está influenciada por los cambios en el caudal del río Apaporis (Ibarra et al. 1979).

\section{Descripción general}

En la Estación Biológica Caparú el bosque primario está muy bien conservado dadas las difíciles condiciones de acceso al lugar (Clavijo-R. 2005) y a que esa zona es considerada como reserva forestal por los miembros del resguardo mencionado. Esto ha permitido la ejecución de estudios científicos enfocados en describir los patrones ecológicos de un bosque prístino.

La estación se sitúa sobre una terraza de origen pleistocénico y su territorio hace parte de la transición biogeográfica de la región amazónica y la región de la Guyana. Se presentan afloramientos rocosos de origen precámbrico, como la Serranía de Taraira, y formaciones terciarias sobre rocas sedimentarias (Defler 1991).

En general, los suelos son superficiales, ácidos, de baja fertilidad y textura arcillosa. Dominan suelos minerales de baja evolución, como Entisoles e Inceptisoles, y suelos de alto grado de evolución, como Ultisoles y Oxisoles. Alrededor del lago Taraira, en la zona inundable, se encuentran suelos poco evolucionados, inundables y predominantemente mal drenados (Ibarra et al. 1979), con pH entre 4.8 y 5.0 (Defler \& Defler 1996).

El clima en la estación es cálido súper húmedo, con un régimen de lluvias bimodal, ligeramente estacional (Rangel \& Luengas 1997). La precipitación anual es de 4000 mm (Defler 1996). Generalmente, el mes que presenta máximas precipitaciones es mayo $(386.3 \mathrm{~mm})$ mientras que el mes más seco suele ser septiembre $(258 \mathrm{~mm})$ (Palacios-Acevedo \& Rodríguez-Rodríguez 1995). Las lluvias están 
distribuidas de manera relativamente homogénea a lo largo del año y no se presenta una estación seca marcada (González 2006). La temperatura media anual es de $25.1^{\circ} \mathrm{C}$, con leves variaciones a lo largo del año (Defler 1996).

\section{Estructura florística}

En el área de estudio se han descrito cinco tipos de bosque: Terraza, Colina, Igapó, Transición y Bajo de caño (Defler \& Defler 1996).

El bosque de Colina se caracteriza por la presencia de pequeñas colinas y es el más abundante en el área de estudio. El dosel es de $25 \mathrm{~m}$ de altura en promedio, se presentan algunos árboles emergentes y el sotobosque es un poco más denso que en el bosque de Terraza y mucho más que en el de Igapó. Entre las familias botánicas más ricas se destacan Moraceae y Fabaceae s.l. (Defler \& Defler 1996).

Por su parte, el bosque de Terraza se caracteriza por ser relativamente llano sin presentar inundaciones periódicas, pues se encuentra $7 \mathrm{~m}$ por encima del nivel máximo de inundación del lago Taraira (Defler 1989). El dosel en Terraza tiene en promedio 30 $\mathrm{m}$ de alto. Según Mantilla-Meluk \& Barrios-Rodríguez (1999), las especies de árboles dominantes de este bosque son Oenocarpus bataua (Arecaceae) y Micrandra spruceana (Euphorbiaceae).

El bosque de Igapó es inundado anualmente por las aguas negras del lago Taraira. La superficie del terreno es relativamente plana, el dosel es considerablemente más bajo que en los bosques de Colina y Terraza, y el sotobosque es menos denso. Según Cárdenas et al. (1997) las principales especies de este bosque son Ormosia coccinaea y Monopteryx иаиси (Fabaceae), Eschweilera coriacea y E. punctata (Lecythidaceae) y Erisma japura (Vochysiaceae).

\section{FASE DE CAMPO}

Entre los meses de diciembre de 2007 y junio de 2008 se establecieron tres parcelas de una ha, una en Colina, una en Terraza y una en Igapó. La elección de la zona exacta en la que se delimitaría cada parcela se realizó aleatoriamente, de tal manera que quedaran relativamente cerca de la estación y en cuadrantes del sistema de trochas que incluyeran un solo tipo de bosque.

Posterior a la delimitación de las parcelas, se marcaron todas las plantas cuyo diámetro a la altura del pecho (DAP) fuera mayor o igual a $10 \mathrm{~cm}$ (CAP $\geq 31$ $\mathrm{cm}$ ). Esta categoría incluyó árboles, palmas y bejucos. Después se procedió a recolectar una muestra de cada planta marcada para su posterior identificación taxonómica. Además se midió el DAP de cada individuo y se registró la presencia o ausencia de exudados o aromas en su corteza. Se llevó a cabo un proceso de identificación preliminar del material botánico en campo y se asignaron morfo-tipos a cada individuo.

\section{FASE POSTERIOR AL TRABAJO DE CAMPO}

La determinación del material botánico se realizó en el Laboratorio de Ecología de Bosques Tropicales y Primatología de la Universidad de los Andes, en el Herbario Amazónico (COAH) y en el Herbario Forestal de la Universidad Distrital (UDBC).

\section{Análisis de datos}

El análisis de los datos se dividió en tres secciones. La primera contiene generalidades de la estructura de los bosques estudiados, la segunda pretende cuantificar de diferentes formas la diversidad local y comparar las tres parcelas en términos de diversidad, y la tercera se ocupa de describir la composición florística de cada parcela y comparar florísticamente las tres parcelas en conjunto. A continuación se explica cómo se analizaron los datos según las secciones mencionadas.

\section{Diversidad}

Para estimar la diversidad se calcularon tres índices: 1. Riqueza de especies: número de especies en cada parcela. 2. Proporción de especies por tallo: una medida de diversidad en la que se corrige la riqueza por el número de individuos, para asegurar que la diversidad que se está calculando no se dé por efecto de la cantidad de individuos del área muestreada. 3. Índice de diversidad de Fisher $(\alpha$ de Fisher): que permite estimar la diversidad $\alpha$ de una comunidad; entre mayor sea $\alpha$, mayor será la diversidad de ésta (Fisher et al. 1943). 
Además, para comparar la diversidad de las tres comunidades de plantas en conjunto se construyeron tres curvas de rarefracción, una por cada parcela. Una curva de rarefracción es útil cuando se tiene un muestreo de $\mathrm{S}$ especies en una comunidad y se quiere saber cuántas especies se encontraría al hacer un submuestreo en esa comunidad (Krebs 1999). Con esto se pretende comparar la diversidad de los tres tipos de bosque, teniendo en cuenta que una comunidad muy rica presentará un gran número de especies al hacer un submuestreo y que lo contrario ocurriría con una comunidad con pocas especies. Las curvas de rarefracción para cada par- cela fueron construidas con la ayuda del software Estimates 8.0 (Colwell 2006), a partir de una matriz que muestra el número de individuos de cada especie presentes en una subparcela de $20 \times 20 \mathrm{~m}$ (Dallmeier et al. 1992). Con las curvas de rarefracción también se quiere ver, a grandes rasgos, si el esfuerzo de muestreo realizado es suficiente para dar una idea general de la diversidad de los bosques estudiados en la Estación Biológica Caparú.

\section{Composición florística}

Se calculó el Índice de importancia (Íi) (Curtis \& McIntosh 1951):

$$
\dot{I} i=\operatorname{Dominancia}(D)+\text { Densidad }(\rho)+\text { Frecuencia }(F)
$$

Ecuación 1

Para comparar la composición florística entre los diferentes tipos de bosque del área de estudio se construyó una ordenación (MDS), con la que se espera que los puntos más cercanos reflejen similitudes y los más lejanos diferencias florísticas. La ordenación se estableció a partir de una matriz en la que se presentaba el número de individuos de cada especie, presentes en 75 subparcelas de $20 \times 20 \mathrm{~m}$ (en la matriz estaban incluidas todas las especies encontradas en las tres parcelas). Ésta fue transformada en una matriz de "disimilitud" (con base en distancias euclidianas) y con ella se construyó la ordenación. Tanto la matriz de disimilitud como la ordenación se hicieron con la ayuda del software PC-ORD (McCune \& Mefford 2006).

Finalmente se calculó el índice de similaridad de Sørensen (1948) (S), una medida semicuantitativa con la que se puede saber qué tanto se parecen dos comunidades. Entre mayor sea el resultado de este índice, mayor será la similitud de las dos comunidades que se están comparando.

\section{RESULTADOS}

\section{ESTRUCTURA Y GENERALIDADES DE CADA TIPO DE BOSQUE}

Las parcelas de Colina y Terraza presentaron un número similar de individuos (Tabla 1), mientras que la parcela de Igapó presentó cerca de 100 individuos menos. En contraste, el área basal de las tres parcelas es muy similar. El hecho de que la parcela de Igapó presente un número mucho menor de individuos, pero un área basal similar a la de las demás parcelas, se explica porque, en general, la parcela tenía más árboles medianos (entre 30 y 50 cm de DAP) que las demás (Figura 1). La estructura de los tres tipos de bosque, a partir del DAP y del número de individuos hallados en las tres parcelas de 1 ha, indica que los tres bosques presentan una gran cantidad de árboles pequeños $(9.9-30 \mathrm{~cm}$ de DAP), pero el bosque de terraza muestra una frecuencia mucho más elevada de plantas entre los 15.1 y $\operatorname{los} 20 \mathrm{~cm}$ de DAP (Figura 1). Esto se puede entender por la abundancia de palmas de las especies Oenocarpus bataua y, en menor proporción, Euterpe precatoria, cuyos fustes rara vez superan los $20 \mathrm{~cm}$ de DAP.

En el caso de la parcela de Igapó, se observa una tendencia a presentar más árboles medianos que las parcelas de Colina y Terraza, y esto no se debe a la presencia de una especie particular -de hecho un amplio rango de especies compone esta categoría. En las tres parcelas había árboles de hasta 100 cm de DAP, pero la única que presentó árboles más grandes fue la de Colina, con un individuo de la especie Parkia nitida $($ DAP $=119.4 \mathrm{~cm})$ y uno de la especie Buchenavia $\mathrm{cf}$. grandis $(\mathrm{DAP}=152.8 \mathrm{~cm})$. 


\section{DIVERSIDAD}

La parcela del bosque de Colina presentó un número de especies muy superior al de las parcelas de los otros dos bosques (Tabla 1). Para saber si la diferencia tan marcada en el número de especies se debe a un efecto del número de individuos, se calculó el número de especies dividido por el número de tallos, con lo que se comprobó que la parcela de Colina es la más diversa de las tres. El resultado de calcular el Índice $\alpha$ de Fisher también lo confirma y según ese índice la parcela de Colina es casi dos veces más diversa que la de Terraza y cerca de cuatro veces más diversa que la de Igapó (Tabla 1).

Las curvas de rarefracción (Figura 2) confirman una vez más que la parcela de Colina es la que presenta mayor riqueza de especies, seguida por la de Terraza y finalmente por la de Igapó. Adicionalmente, las curvas de rarefracción sugieren que si se hiciera un esfuerzo de muestreo mayor en el bosque de Igapó, no se encontrarían muchas más especies nuevas por unidad de área. Por el contrario, la curva de la parcela de Colina muestra que la cantidad de especies de este tipo de bosque está siendo subestimada y que es necesario un esfuerzo de muestreo mucho mayor para conocer todas las especies que pueden desarrollarse en este hábitat.

La parcela de Colina además fue la más equitativa, según la abundancia relativa de los individuos de todas las especies (Figura 3). Aunque todas las parcelas presentaron especies dominantes, la de Colina es más diversa por la presencia de una gran cantidad de especies raras, mientras que en las otras dos parcelas no hubo un número tan elevado de éstas (Figura 3).

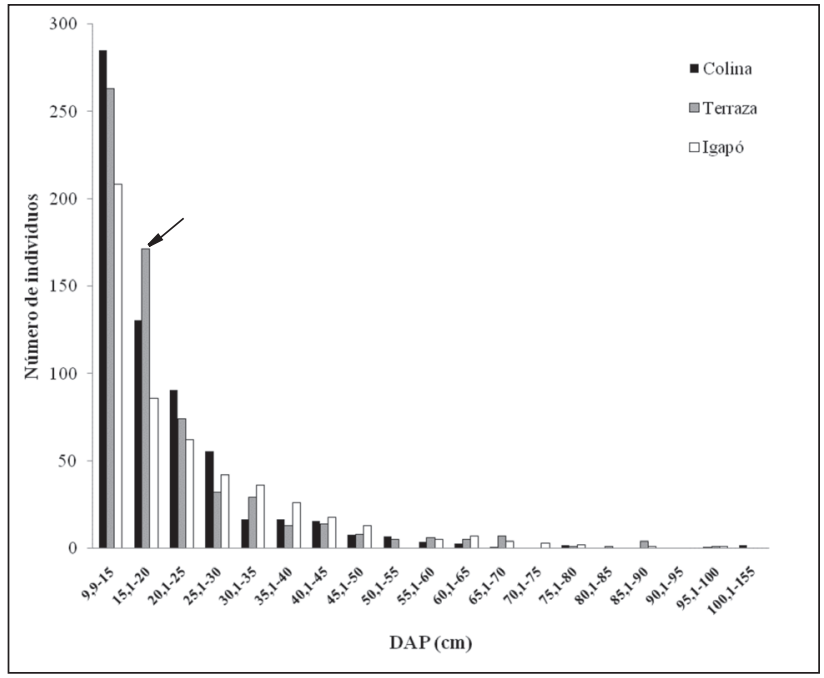

Figura 1. Estructura de la vegetación en la Estación Biológica Caparú, de acuerdo a la frecuencia de individuos en diferentes categorías de DAP en levantamientos de 1 ha en tres tipos de bosque. La flecha indica las diferencias generadas por la alta abundancia de palmas en la parcela de Terraza.

Tabla 1. Resultados generales de la estructura y la diversidad de tres parcelas de vegetación de 1 ha, en diferentes tipos de bosque en la Estación Biológica Caparú.

\begin{tabular}{rccccc}
\hline Parcela & No. individuos & Área basal total $\left(\mathbf{m}^{2}\right)$ & No. sp. (riqueza) & Sp./tallo & a Fisher \\
\hline \hline Colina & 641 & 29.3 & 256 & 0.40 & 160.3 \\
Terraza & 634 & 30.3 & 170 & 0.27 & 78.4 \\
Igapó & 514 & 28.6 & 112 & 0.22 & 44.7 \\
\hline
\end{tabular}




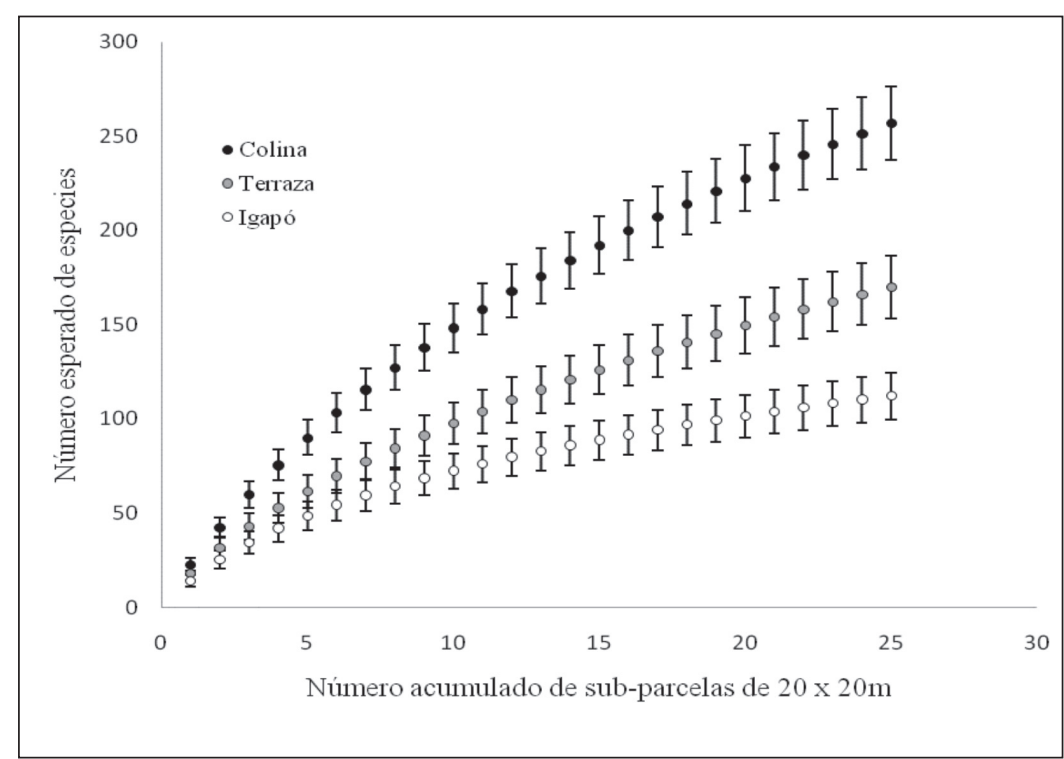

Figura 2. Curvas de rarefracción para comparar la riqueza de especies de tres tipos de bosque en la Estación Biológica Caparú, a partir del levantamientos de vegetación de 1 ha.

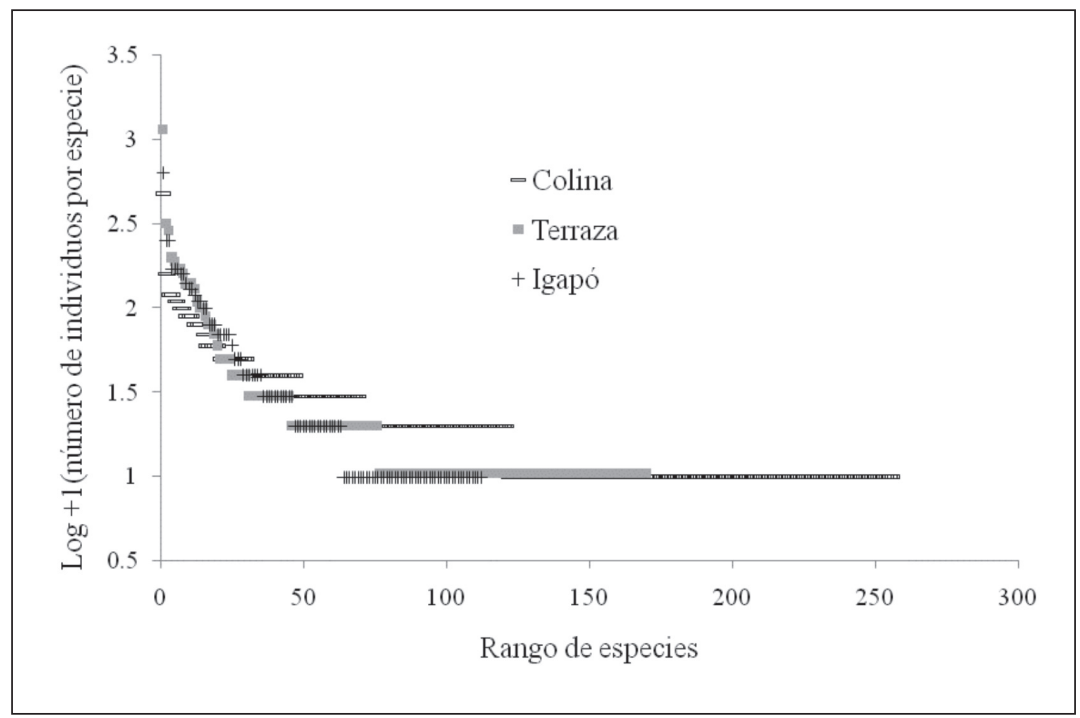

Figura 3. Abundancia relativa de individuos (equitabilidad) para las especies de tres parcelas de bosque en la Estación Biológica Caparú, organizadas según su rango (la primera a la izquierda siendo la más abundante).

\section{COMPOSICIÓN FLORÍSTICA}

Las Tablas 2 y 3 muestran los resultados del índice de importancia para cada parcela. Las familias $\mathrm{Fa}-$ baceae s.1., Myristicaceae, Euphorbiaceae, Sapotaceae y Lauraceae se encuentran entre las 10 más importantes de los tres tipos de bosque (Tabla 2), lo que sugiere que se trata de taxa exitosos, con especies capaces de colonizar distintos ambientes. Estas familias no siempre están representadas por las mismas especies en las tres parcelas, sin embar- 
go hay casos en los que las mismas especies de una familia se encuentran entre las 15 más importantes de dos parcelas. Dos ejemplos son las familias Fabaceae s.1. y Myristicaceae, que están representadas en Terraza y Colina por las mismas especies: Clathrotropis macrocarpa y Heterostemon ellipticus (Fabaceae s.1.) e Iryanthera ulei y Virola pavonis (Myristicaceae).

Las parcelas de Colina y Terraza también comparten familias importantes como Burseraceae, Moraceae, Arecaceae y Malvaceae s.l., algunas de ellas representadas a su vez por una especie importante: Burseraceae por Protium cf. aracouchini, Moraceae por Brosimum rubescens y Arecaceae por $E u$ terpe precatoria.

La familia Lecythidaceae se encuentra entre las 10 más importantes de las parcelas de Colina e Igapó, pero está representada en la primera por Eschweilera coriacea, que de hecho es la especie más importante de esa parcela (Tabla 3), y en la segunda por E. albiflora y E. parvifolia. Esto sugiere que hay familias capaces de colonizar hábitats muy distintos cuando las especies que las conforman se especializan para sobrevivir en alguno de ellos. Otro ejemplo de ello es la familia Clusiaceae, que se encuentra entre las 10 más importantes de Terraza e Igapó (Tabla 2).

En este caso particular la especie Caraipa punctulata es la quinta más importante de la parcela de Terraza, mientras que Caraipa densifolia es la especie más importante de la parcela de Igapó (Tabla 3).

Aunque las familias Apocynaceae y Chrysobalanaceae se encuentran en las tres parcelas, sólo hacen parte de las 10 más importantes de la parcela de Igapó (Tabla 2). En el caso de Apocynaceae esto se debe a la presencia de la especie Aspidosperma excelsum (Tabla 3). En cuanto a la familia Chrysobalanaceae, el hecho de que se encuentre en la lista de las 10 familias más importantes de Igapó se debe a la presencia de muchas especies distintas en ese tipo de bosque (Licania micrantha, L. longistyla, L. octandra, L. licaniaeflora, L. vaupesiana, Couepia paraensis, $C$. cf. doliochopoda y $C$. cf. racemosa).

En la parcela de Colina la especie más importante supera en gran medida a las siguientes en la escala de clasificación (Tabla 3). Eschweilera coriacea no sólo presenta un índice de importancia dos veces mayor que el de la siguiente especie, sino que además la supera claramente en los tres componentes de dicho índice. En las demás parcelas la diferencia entre la especie más importante y las siguientes no es tan marcada.

Para comparar la composición florística de las tres hectáreas muestreadas se realizó una ordenación en dos dimensiones. En la Figura 4 se observa que las 25 subparcelas de cada bosque se encuentran relativamente agrupadas en una sección del espacio bidimensional. Las subparcelas situadas en las zonas más extremas de cada grupo son las que contenían un gran número de individuos de la especie más importante de cada bosque. Así, los puntos grises más cercanos al eje 2 de la Figura 4 representan subparcelas del bosque de Terraza con nueve u ocho individuos de Oenocarpus bataua, especie que presentó una relación fuerte con el eje $1(\mathrm{r}=-0.83)$. Igualmente, los puntos negros de la sección superior central simbolizan subparcelas del bosque de Colina que contienen cinco individuos de la especie Eschweilera coriacea. Esta especie de la familia Lecythidaceae presentó una relación positiva fuerte con el eje $2(r=0.66)$. Por último, los puntos blancos de la esquina inferior derecha son subparcelas del bosque de Igapó que contenían hasta ocho individuos de la especie Caraipa densifolia, que presentó una correlación fuerte con el eje $1(\mathrm{r}=0.72$, Figura 4). 
Tabla 2. Listado de las 10 familias de plantas más importantes en una parcela de 1 ha, en tres tipos de bosque de la Estación Biológica Caparú, donde: $\mathrm{D}=$ Dominancia, $\rho=$ densidad, $\mathrm{F}=$ frecuencia.

\begin{tabular}{|c|c|c|c|c|c|c|}
\hline & No. & Familia & D & $\rho$ & $\mathbf{F}$ & Índice de importancia \\
\hline \multirow{10}{*}{ 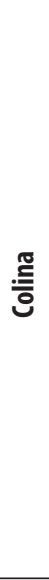 } & 1 & FABACEAE s.l. & 19.2 & 15.0 & 92 & 126.1 \\
\hline & 2 & MORACEAE & 12.0 & 10.9 & 96 & 118.9 \\
\hline & 3 & LECYTHIDACEAE & 8.6 & 10.1 & 88 & 106.8 \\
\hline & 4 & BURSERACEAE & 6.0 & 8.9 & 88 & 102.9 \\
\hline & 5 & MYRISTICACEAE & 5.6 & 7.8 & 84 & 97.4 \\
\hline & 6 & EUPHORBIACEAE & 5.7 & 6.2 & 80 & 91.9 \\
\hline & 7 & SAPOTACEAE & 6.4 & 5.8 & 76 & 88.2 \\
\hline & 8 & ARECACEAE & 2.1 & 3.6 & 64 & 69.7 \\
\hline & 9 & MALVACEAE s.l. & 3.7 & 3.7 & 56 & 63.5 \\
\hline & 10 & LAURACEAE & 2.7 & 3.7 & 56 & 62.5 \\
\hline \multirow{10}{*}{ 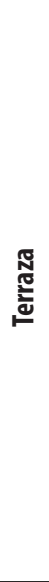 } & 1 & ARECACEAE & 9.4 & 22.9 & 100 & 132.3 \\
\hline & 2 & FABACEAE s.l. & 25.3 & 14.2 & 92 & 131.5 \\
\hline & 3 & EUPHORBIACEAE & 15.4 & 11.2 & 88 & 114.6 \\
\hline & 4 & MYRISTICACEAE & 5.5 & 9.1 & 80 & 94.7 \\
\hline & 5 & MORACEAE & 7.6 & 6.2 & 80 & 93.7 \\
\hline & 6 & BURSERACEAE & 3.0 & 5.2 & 68 & 76.3 \\
\hline & 7 & CLUSIACEAE & 3.0 & 3.5 & 60 & 66.5 \\
\hline & 8 & LAURACEAE & 1.4 & 2.7 & 56 & 60.1 \\
\hline & 9 & SAPOTACEAE & 3.2 & 3.3 & 48 & 54.5 \\
\hline & 10 & MALVACEAE s.l. & 3.8 & 2.5 & 48 & 54.3 \\
\hline \multirow{10}{*}{ 응 } & 1 & FABACEAE s.l. & 37.1 & 30.4 & 100 & 167.4 \\
\hline & 2 & LECYTHIDACEAE & 12.0 & 7.0 & 76 & 95.0 \\
\hline & 3 & CLUSIACEAE & 9.2 & 12.6 & 72 & 93.8 \\
\hline & 4 & CHRYSOBALANACEAE & 6.2 & 9.1 & 72 & 87.3 \\
\hline & 5 & EUPHORBIACEAE & 3.4 & 5.4 & 60 & 68.9 \\
\hline & 6 & APOCYNACEAE & 2.7 & 4.1 & 52 & 58.8 \\
\hline & 7 & MYRISTICACEAE & 1.6 & 5.1 & 48 & 54.6 \\
\hline & 8 & SAPOTACEAE & 4.4 & 3.1 & 44 & 51.5 \\
\hline & 9 & LAURACEAE & 2.4 & 2.5 & 40 & 45.0 \\
\hline & 10 & ANNONACEAE & 1.4 & 3.5 & 40 & 44.9 \\
\hline
\end{tabular}


Tabla 3. Listado de las 15 especies de plantas más importantes en una parcela de 1 ha, en tres tipos de bosque de la Estación Biológica Caparú, donde: $\mathrm{D}=$ Dominancia, $\rho=$ densidad, $\mathrm{F}=$ frecuencia.

\begin{tabular}{|c|c|c|c|c|c|c|}
\hline & No. & Especie & D & $\rho$ & $\mathrm{F}$ & Índice de importancia \\
\hline \multirow{15}{*}{ 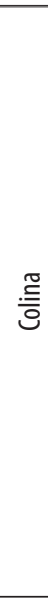 } & 1 & Eschweilera coriacea & 6.6 & 7.5 & 88 & 102.1 \\
\hline & 2 & Protium cf. aracouchini & 1.3 & 2.5 & 48 & 51.8 \\
\hline & 3 & Iryanthera ulei & 0.7 & 1.9 & 40 & 42.6 \\
\hline & 4 & Micropholis venulosa & 1.8 & 1.6 & 36 & 39.4 \\
\hline & 5 & Iriartea deltoidea & 1.2 & 1.7 & 36 & 39.0 \\
\hline & 6 & Brosimum lactescens & 1.9 & 1.3 & 32 & 35.2 \\
\hline & 7 & Clathrotropis macrocarpa & 1.1 & 1.9 & 32 & 35.0 \\
\hline & 8 & Rinorea paniculata & 1.2 & 1.7 & 32 & 34.9 \\
\hline & 9 & Pseudolmedia laevis & 0.9 & 1.2 & 32 & 34.1 \\
\hline & 10 & Euterpe precatoria & 0.6 & 1.6 & 32 & 34.1 \\
\hline & 11 & Heterostemon ellipticus & 0.4 & 1.4 & 32 & 33.8 \\
\hline & 12 & Aldina heterophylla & 0.6 & 1.4 & 28 & 30.0 \\
\hline & 13 & Brosimum rubescens & 0.5 & 1.1 & 28 & 29.6 \\
\hline & 14 & Cynometra cf. Iongicuspis & 1.9 & 1.3 & 24 & 27.1 \\
\hline & 15 & Virola pavonis & 1.5 & 1.4 & 24 & 26.9 \\
\hline \multirow{15}{*}{ 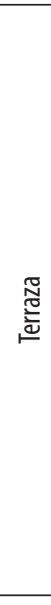 } & 1 & Oenocarpus bataua & 8.2 & 18.1 & 100 & 126.3 \\
\hline & 2 & Micrandra spruceana & 10.3 & 5.0 & 76 & 91.3 \\
\hline & 3 & Monopteryx uаuси & 14.9 & 3.0 & 56 & 73.9 \\
\hline & 4 & Euterpe precatoria & 1.1 & 4.6 & 60 & 65.7 \\
\hline & 5 & Caraipa punctulata & 2.2 & 2.7 & 48 & 52.8 \\
\hline & 6 & Brosimum rubescens & 3.9 & 2.5 & 44 & 50.4 \\
\hline & 7 & Protium cf. aracouchini & 1.7 & 2.7 & 44 & 48.4 \\
\hline & 8 & Sandwithia heterocalyx & 0.9 & 3.2 & 44 & 48.1 \\
\hline & 9 & Iryanthera ulei & 0.7 & 2.2 & 44 & 46.9 \\
\hline & 10 & Virola pavonis & 2.4 & 2.2 & 40 & 44.6 \\
\hline & 11 & Iryanthera crassifolia & 0.9 & 2.2 & 40 & 43.1 \\
\hline & 12 & Scleronema micranthum & 2.3 & 1.6 & 36 & 39.9 \\
\hline & 13 & Clathrotropis macrocarpa & 0.5 & 2.1 & 36 & 38.6 \\
\hline & 14 & Brosimum utile & 2.9 & 1.7 & 32 & 36.6 \\
\hline & 15 & Hevea cf. guianensis & 2.4 & 1.3 & 32 & 35.6 \\
\hline \multirow{15}{*}{ 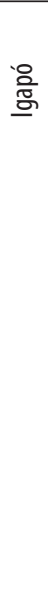 } & 1 & Caraipa densifolia & 8.8 & 12.3 & 68 & 89.1 \\
\hline & 2 & Zygia cataractae & 2.8 & 4.9 & 56 & 63.7 \\
\hline & 3 & Aldina heterophylla & 3.8 & 3.1 & 44 & 50.9 \\
\hline & 4 & Virola elongata & 1.4 & 4.9 & 44 & 50.3 \\
\hline & 5 & Dicorynia cf. paraensis & 6.7 & 3.3 & 36 & 46.0 \\
\hline & 6 & Acosmium nitens & 4.6 & 2.1 & 36 & 42.7 \\
\hline & 7 & Buchenavia viridiflora & 7.3 & 3.1 & 32 & 42.4 \\
\hline & 8 & Aspidosperma excelsum & 2.5 & 3.3 & 36 & 41.8 \\
\hline & 9 & Cynometra marginata & 2.0 & 2.7 & 36 & 40.7 \\
\hline & 10 & Crudia oblonga & 1.7 & 2.5 & 36 & 40.2 \\
\hline & 11 & Copaifera officinalis & 1.7 & 2.1 & 36 & 39.9 \\
\hline & 12 & Eschweilera albiflora & 4.5 & 1.6 & 32 & 38.1 \\
\hline & 13 & Licania micrantha & 2.8 & 1.9 & 32 & 36.7 \\
\hline & 14 & Eschweilera parvifolia & 1.4 & 2.3 & 32 & 35.7 \\
\hline & 15 & Mabea nitida & 1.3 & 1.9 & 32 & 35.2 \\
\hline
\end{tabular}

El índice de similaridad de Sørensen indicó que las parcelas más parecidas son las de Terraza y Colina (Tabla 4). La comparación individual de cada una de esas parcelas con la de Igapó muestra que ambas se diferencian de esta última en igual medida. 


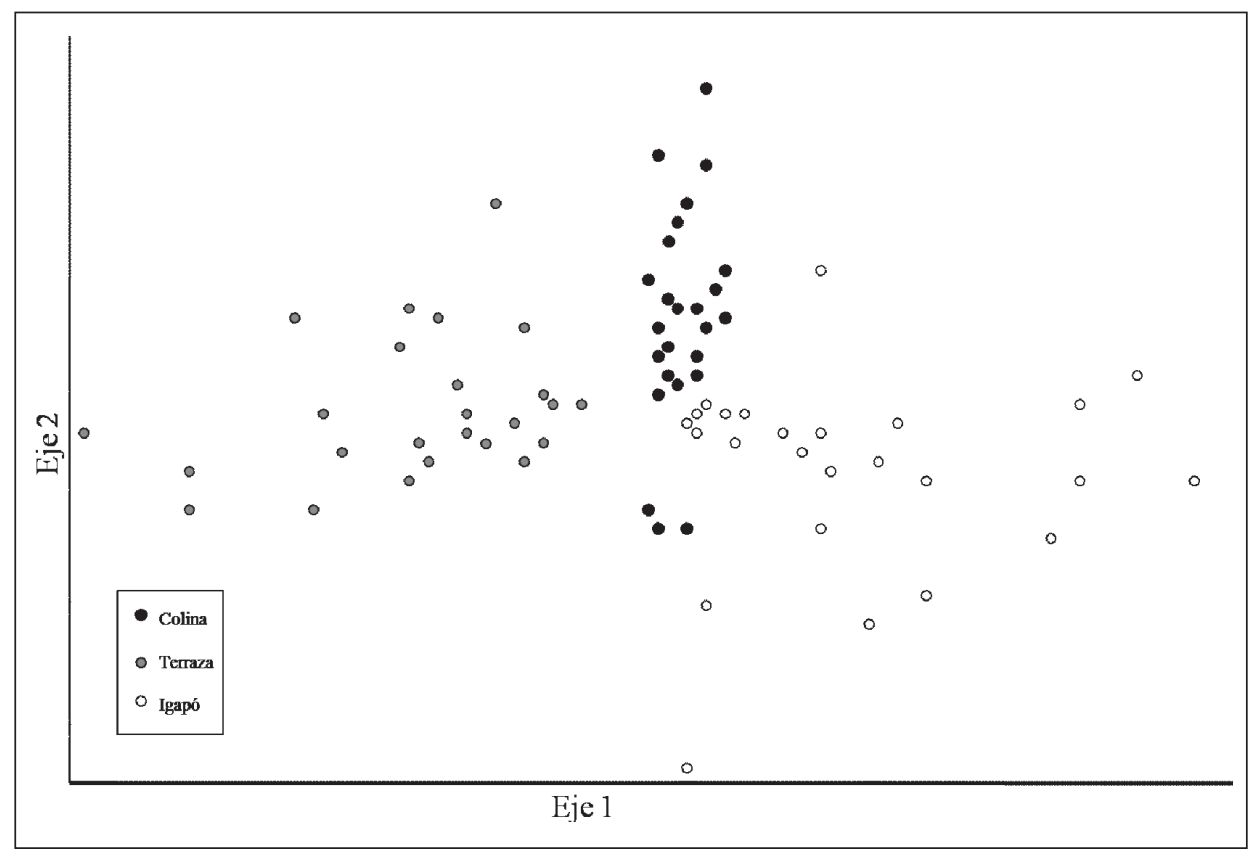

Figura 4. Ordenación de 75 subparcelas $(20$ × 20 m) de vegetación en tres tipos de bosque (Colina, Terraza e Igapó) en la Estación Biológica Caparú, a partir de una matriz de abundancia de especies.

Tabla 4. Comparación de tres parcelas de vegetación en la Estación Biológica Caparú utilizando el índice de similaridad de Sørensen (Ss), donde A es el número de especies compartidas por las dos parcelas que se estén comparando, b es el número de especies presentes en la parcela $\mathrm{B}$ y no en la parcela $\mathrm{C}$, y $\mathrm{c}$ es el número de especies presentes en la parcela $\mathrm{C}$ y no en la parcela $\mathrm{B}$.

\begin{tabular}{cccc}
\hline $\begin{array}{c}\text { Comparación } \\
\text { (B vs. C) }\end{array}$ & A & b;c & Ss \\
\hline Colina vs. Terraza & 76 & $180 ; 94$ & 0.36 \\
Colina vs. Igapó & 21 & $236 ; 91$ & 0.11 \\
Terraza vs. Igapó & 15 & $155 ; 97$ & 0.11 \\
\hline
\end{tabular}

\section{DISCUSIÓN DE RESULTADOS}

\section{DIVERSIDAD}

Con base en estimaciones previas realizadas por Defler \& Defler en 1996 se planteó la hipótesis de que el bosque de Colina es el más diverso, seguido por el de Terraza, y éste a su vez por el de Igapó.
Según los resultados incluidos en la Tabla 1, todas las medidas de diversidad confirman esa hipótesis y las observaciones de Defler \& Defler. La Figura 2 y los resultados de calcular el alfa de Fisher (Tabla 1) no sólo soportan lo dicho, sino que además dan una idea de qué tan diferente es la diversidad entre los tres tipos de bosque. Estos resultados también confirman la hipótesis que proponía que el bosque de Igapó es considerablemente distinto a los otros dos tipos de bosque, pues en términos de diversidad se mostró que es muy inferior a los bosques de Colina y Terraza.

La parcela de Colina se encuentra entre las más diversas de la Amazonía colombiana, mientras que la parcela de Terraza se halla en un nivel intermedio (Figura 5). Por su parte, la parcela de Igapó presenta una diversidad mayor que la de otros bosques inundables de la Amazonía como son los estudiados por Stevenson et al. (2004) en el Parque Nacional Natural Tinigua. Sin embargo es importante aclarar que dichos bosques inundables son várzeas, es decir, son inundados por ríos de aguas blancas, lo que implica que las condiciones de ambos tipos de bosque son diferentes. 


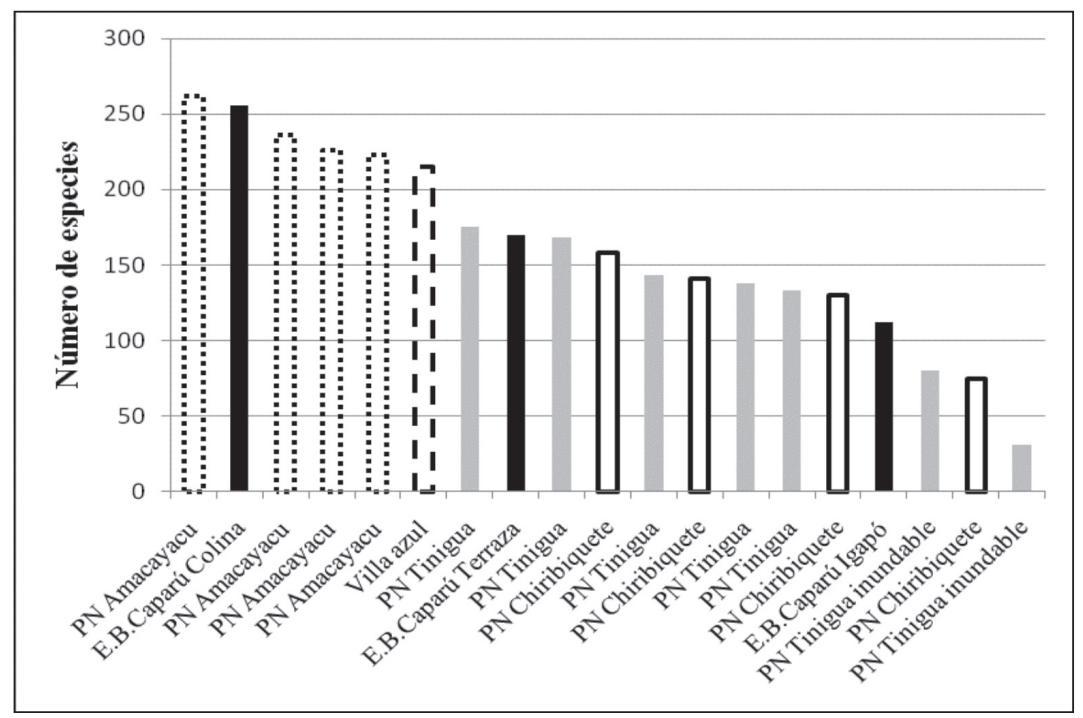

Figura 5. Comparación de la riqueza de especies de diferentes parcelas de vegetación de una hectárea (DAP $\geq 10 \mathrm{~cm}$ ) en la Amazonía colombiana (construida a partir de los estudios de Stevenson et al. 2004 y Duque et al. 2007 en revisión).

En 2000, Ter Steege y colaboradores hicieron un estudio de la diversidad y la composición florística de la Amazonía (incluyendo la zona del Escudo Guyanés). Tomando la información de 94 parcelas de vegetación de una ha (70 en bosques de Terra Firme (TF) y 24 en planos inundables (FL); árboles de 10 o más cm de DAP), calcularon un alfa de Fisher promedio para TF y uno para FL, para cuatro zonas geográficas de la Amazonía (Tabla 5).

Aunque Ter Steege et al. (2000) no definieron un límite para cada zona de la Amazonía, la Estación Biológica Caparú se encontraría situada en una zona intermedia entre la Amazonía occidental y la Amazonía central. La diversidad calculada para la parcela del bosque de Colina ( $\alpha$ de Fisher $=160.3$, Tabla 1) es muy superior a la diversidad promedio en bosques de Terra Firme, tanto de la Amazonía occidental como de la Amazonía central (Tabla 5), lo que implica que dicho tipo de bosque en la Estación Biológica Caparú se encuentra entre los más diversos de la Amazonía. Por el contrario, la diversidad de la parcela de Terraza $(\alpha$ de Fisher $=$ 78.4) se encuentra por debajo de ambos promedios, por lo que se puede inferir que es un bosque relativamente poco diverso. Promediando la diversidad de la parcela de Colina con la de Terraza ( $\alpha$ de Fisher $=119.3$ ) se obtiene un dato de diversidad cercano a los promedios calculados por Ter Steege et al. (2000) (Tabla 5) para los bosques de Terra Firme en la Amazonía occidental y central. Esto sugiere que los bosques de Terra Firme de la Estación Biológica Caparú presentan una diversidad intermedia, si son comparados con otros bosques de la Amazonía occidental y central, y alta, si son comparados con los de la Amazonía oriental y el Escudo Guyanés.

Tabla 5. Diversidad alfa promedio para parcelas de 1 ha en el núcleo del bosque húmedo de la Amazonía (core Amazonian rain forest: $8^{\circ} \mathrm{S}=8^{\circ} \mathrm{N}$, precipitación anual $\geq 2000 \mathrm{~mm}$ ). $\mathbf{P}$ indica significancia para una ANOVA de una vía por tipo de bosque (tabla modificada de Ter Steege et al. 2000).

\begin{tabular}{ccc}
\hline Bosque & Tierra firme & Inundados \\
\hline Amazonía occidental & $124.5(221.1)$ & $78.7(131.7)$ \\
Amazonía central & $1263(221.8)$ & $46.1(109.4)$ \\
Amazonía oriental & $56.0(123.6)$ & $20.8(24.6)$ \\
Escudo Guyanés & $51.9(155.5)$ & $16.7(22.7)$ \\
$P$ & $<0.01$ & $<0.01$ \\
\hline
\end{tabular}

La diversidad calculada para el bosque de Igapó ( $\alpha$ de Fisher $=44.7$, Tabla 1 ) es inferior a la diversidad promedio en bosques inundables de la Amazonía occidental (Tabla 5), y muy cercana a la de los de la Amazonía central, de lo que se concluye que el bosque de Igapó de la Estación Biológica Caparú pre- 
senta una diversidad intermedia en relación a otros bosques inundables de la Amazonía en general. La diversidad de los bosques de la Estación Biológica Caparú es entonces similar a la de los bosques de la Amazonía occidental y central. Sin embargo, sería necesario tener un mayor esfuerzo de muestreo para llegar a conclusiones más robustas, dado que en un solo lugar amazónico la diversidad puntual puede variar mucho (Stropp et al. 2009).

Comparando los bosques de Terra Firme con el inundable, es posible que las condiciones hostiles del segundo (por ejemplo, periodos prolongados de inundación) limiten el número de especies que pueden establecerse en el bosque de Igapó y únicamente las especies adaptadas a esas condiciones logren sobrevivir a la inundación anual (Clinebe11 et al. 1995, Duivenvoorden 1996, Parolin et al. 2004b). Ter Steege et al. (2000) hallaron el mismo patrón de diversidad para toda la Amazonía, concluyendo que los bosques inundables son sistemáticamente menos diversos que los de Terra Firme.

Pero ¿cómo se explica la diferencia de diversidad entre los bosques de Colina y Terraza, dado que ambos son no inundables y se encuentran a distancias geográficas tan cortas? Una posible respuesta sería que factores abióticos como la calidad del suelo, la temperatura y la humedad, operando a escalas geográficas pequeñas, controlen el número y el tipo de especies que pueden establecerse en un tipo de bosque local. Aunque Gentry (1988) mostró que la influencia de la fertilidad de los suelos no era significativa al momento de explicar la diversidad de un lugar, lo hizo a una escala continental. La distancia entre las dos parcelas permanentes de Colina y Terraza es de alrededor de $700 \mathrm{~m}$, lo que quiere decir que aquí se está trabajando con una escala local y quizás dichos factores abióticos sí son influyentes a distancias geográficas tan cortas. Para comprobar si eso es cierto, sería necesario medir la humedad y la temperatura y describir los componentes del suelo de cada tipo de bosque y evaluar si existe una relación entre las restricciones abióticas de cada microhábitat y el tipo de especies presentes en ellos. Adicionalmente, sería importante realizar trasplantes experimentales para verificar las posibilidades de adaptación a cada tipo de suelo.
En la parcela del bosque de Terraza hay 115 individuos de la especie Oenocarpus bataua, 32 de la especie Micrandra spruceana y 29 de la especie Euterpe precatoria. Es decir, el 28\% de todos los individuos de esa parcela pertenecen a tres especies, de las cuales únicamente Euterpe precatoria fue encontrada en la parcela de Colina (con 10 individuos, $1.5 \%$ del los individuos de esa parcela). Entonces se puede suponer que en Terraza la diversidad es menor por la presencia de estas tres especies exitosas, que han logrado ocupar una porción importante de ese bosque. La pregunta entonces es por qué tres especies tan exitosas en Terraza son tan pobremente representadas en Colina. Para responder a esa pregunta son necesarios futuros estudios que evalúen las diferencias abióticas de los bosques de Colina y Terraza y las características de las especies dominantes de cada uno de ellos, para establecer qué condiciones les son favorables (idealmente incluyendo estudios experimentales usando trasplantes).

Vargas (2008), tras estudiar el reclutamiento limitado y su efecto en la diversidad de los tipos de bosque de la Estación Biológica Caparú, halló para plántulas y juveniles el mismo patrón de diversidad para los bosques contemplados aquí, siendo Colina el más diverso e Igapó el menos diverso. Además, encontró tendencias positivas, donde a mayor dispersión de semillas hay mayor diversidad. La explicación de dicho fenómeno, según Vargas, es que en el bosque de Colina la dispersión de semillas es más efectiva y por eso hay mayor número de especies representadas. Las propuestas enunciadas anteriormente no dejan de ser aproximaciones no excluyentes para responder al por qué de la gran diferencia de diversidad entre los bosques de $T e$ rra Firme estudiados aquí, pero la pregunta sigue abierta y son necesarios más estudios para responderla.

\section{COMPOSICIÓN FLORÍSTICA}

Según reportes previos (Defler \& Defler 1996, Cárdenas et al. 1997, Mantilla-Meluk \& BarriosRodríguez 1999, Gonzalez 2007), se esperaba que los bosques estudiados aquí estuvieran compuestos principalmente por especies de las familias Areca- 
ceae, Combretaceae, Euphorbiaceae, Fabaceae s.1., Lecythidaceae, Moraceae, Myristicaceae, Sapotaceae, Urticaceae s.l. y Vochysiaceae. Las familias y especies más importantes de cada bosque están listadas en las Tablas 2 y 3 respectivamente. Los resultados confirman que algunas de las familias propuestas en los reportes previos como importantes, lo son.

Por estar presentes en la lista de las 10 familias más importantes de los tres tipos de bosque, las familias Euphorbiaceae, Fabaceae s.l., Lauraceae, Myristicaceae y Sapotaceae se consideran las más importantes en el área de estudio. Otras familias importantes, compartidas por dos tipos de bosque, fueron Arecaceae, Burseraceae, Malvaceae s.l. y Moraceae, en Colina y Terraza; Lecythidaceae, en Colina e Igapó, y Clusiaceae, en (Terraza e Igapó. Contrario a lo esperado, las familias Combretaceae, Urticaceae s.l. y Vochysiaceae no estuvieron presentes en la lista de las más importantes de ninguno de los tipos de bosque.

La familia más importante en los tres tipos de bosque fue Fabaceae s.l. Aunque en el bosque de Colina Arecaceae figura como la más importante, la diferencia entre esta familia de palmas con Fabaceae s.l. es baja (Tabla 2). Este resultado coincide con lo planteado por Gentry (1988, 1992), quien determinó que los bosques húmedos tropicales a nivel mundial son consistentemente dominados por un pequeño grupo de familias encabezado por las leguminosas. Ter Steege et al. (2000) también confirmaron el planteamiento de Gentry, al hallar que el 16\% de los individuos en 94 parcelas de 1 ha $(\mathrm{DAP} \geq 10 \mathrm{~cm})$ en toda la Amazonía pertenecen a dicha familia.

Terborgh \& Andresen (1998) analizaron la composición del bosque amazónico a escalas locales y regionales. Entre sus resultados determinaron que cerca del $80 \%$ de los árboles (DAP $\geq 10 \mathrm{~cm}$ ) de la Amazonía pertenecen a las 16 familias que se listan a continuación: Annonaceae, Arecaceae, Bombacaceae, Burseraceae, Chrysobalanaceae, Euphorbiaceae, Fabaceae s.1., Lauraceae, Lecythidaceae, Meliaceae, Moraceae, Myristicaceae, Rubiaceae, Sapotaceae, Sterculiaceae y Violaceae. Este estu- dio coincide con lo expuesto por Terborgh \& Andresen (1998), pues en las parcelas levantadas se encontraron individuos pertenecientes a todas esas familias y porque muy pocas de ellas (Meliaceae, Rubiaceae y Violaceae) no hacen parte de la lista de las 10 más importantes de alguno de los tres bosques contemplados.

Aunque los tres bosques comparten muchas familias importantes, y a veces lo mismo ocurre con algunas especies, la composición florística de cada uno demuestra que se trata de unidades del paisaje distintas unas de otras. De un lado, las especies más importantes de cada bosque permiten separarlos florísticamente (Figura 4) y, de otro lado, son muchos los casos en los que una especie sólo se encontró en un tipo de bosque. Quizás el caso más claro de ello es el del bosque de Igapó, que es considerablemente distinto a los bosques de Terra Firme. La comparación realizada mediante el Índice de Similaridad de Sørensen (Tabla 4) demuestra que florísticamente los bosques no inundables se parecen más entre sí que con el de Igapó. Además, familias como Annonaceae, Apocynaceae y Chrysobalanaceae, que estuvieron presentes entre las más importantes del plano de inundación, fueron mucho menos dominantes en los otros dos bosques. Entonces se responde otra pregunta planteada por este estudio, pues así como se había demostrado que en términos de diversidad los tres tipos de bosque diferían entre sí, se puede afirmar lo mismo desde un punto de vista florístico.

\section{CONCLUSIONES}

En la Estación Biológica Caparú el bosque de Colina es el más diverso, seguido por el de Terraza y después por el de Igapó. Lo anterior se concluye a partir de los resultados de este estudio, de las estimaciones previas realizadas por Defler \& Defler (1996) y de los patrones de diversidad de semillas dispersadas y plántulas estimados por Vargas (2008). Por su dominancia, frecuencia y densidad, las familias de plantas de dosel más importantes son Fabaceae s.l., Sapotaceae, Euphorbiaceae, Lauraceae y Myristicaceae y esto concuerda con lo que se ha reportado para los bosques amazónicos. Tanto en su diversidad como en su composi- 
ción florística, los tres tipos de bosque estudiados presentan grandes diferencias. Si bien el bosque de Igapó es el más disímil de los tres, los bosques de Terra Firme muestran diferencias marcadas y la explicación a este fenómeno requiere de futuros estudios. En general, la diversidad estimada para los bosques del área de estudio es alta con respecto a la de otros bosques de la Amazonía colombiana y a la de bosques de zonas más orientales de la cuenca amazónica. Por ser una zona de bosque primario poco intervenido y presentar niveles tan altos de diversidad, el área de estudio debería mantener su estatus de reserva forestal para promover su conservación.

\section{AGRADECIMIENTOS}

Este estudio no hubiera sido posible sin la colaboración de los asistentes de campo que nos ayudaron a colectar muestras botánicas: Óscar Iván Yucuna, Luis Barasano, Alfredo Chávez y Luis Tanimuca. Por su valiosa colaboración en el procesamiento del material botánico agradecemos al Laboratorio de Bosques Tropicales y Primatología y al Herbario Amazónico (COAH), especialmente a Dairon Cárdenas. Por el apoyo logístico y emocional, queremos agradecer a Marcos González, Vera Schütz, Nicolás Rodríguez, Sergio Botero, Juan Piña y demás trabajadores de la estación. La financiación de este proyecto provino de recursos personales, del Laboratorio de Bosques Tropicales y Primatología, del Herbario Amazónico (COAH) y de Conservación Internacional. Ivonne Natalia Vargas aportó los tubos para las parcelas.

\section{REFERENCIAS BIBLIOGRÁFICAS}

Bennet-Defler, S. 1994. Las aves de la Estación Caparú: Una lista preliminar de especies. Trianea 5: 379-400.

Burnham, R. J. 2004. Alpha and beta diversity of lianas in Yasuni, Ecuador. Forest Ecology and Management 86: 975-978.

Cárdenas, D., D. Giraldo-Cañas \& C. Arias. 1997. Vegetación. En: IGAC (ed.). Zonificación ambiental para el plan modelo colombo-brasilero (eje Apaporis-Tabatinga: PAT). Editorial Linotipia Bolivar. Bogotá. Pp. 183-228.
Carvajal-L., F. J., F. N. Posada-A., L. C. MolinaM., A. Delgado-F., L. E. Acero-D., O. Araújo-M. \& F. Rodríguez-M. 1979. Bosques. En: Proyecto Radargramétrico del Amazonas (ed.). La Amazonia colombiana y sus recursos. Bogotá. Pp. 217-322.

Clavijo-R., L. 2005. Las lianas de la estación biológica Mosiro Itajura (Caparú), bajo río Apaporis (Vaupés Colombia). Tesis de pregrado. Departamento de Biología, Facultad de Ciencias, Universidad Nacional de Colombia. Bogotá.

Clinebell, R. R., O. L. Phillips, A. H. Gentry, N. Stark \& H. Zuuring. 1995. Prediction of neotropical tree and liana species richness from soil and climatic data. Biodiversity and Conservation 4: 56-90.

Colinvaux, P. A. \& P. E. De Oliveira. 2001. Amazon plant diversty and climate through the Cenozoic. Paleography, Paleoclimatology, Paleoecology 166: 51-63.

Colwell, R. K. 2006. Estimate S: Statistical estimation of species richness and shared species from samples. Versión 8.0.

Curtis, J. T. \& R. P. Mcintosh. 1951. An upland forest continuum in the prairie-forestborder region of Wisconsin. Ecology 32: 476-496.

Dallmeier, F., R. B. Foster, C. B. Romano, R. Rice \& M. Kabel. 1992. Guía para el usuario de las parecelas experimentales de biodiversidad. Reserva de la Biosfera del Beni, Bolivia. Institución Smithsonian, Programa del Hombre y la Biosfera. La Paz.

Defler, T. R. 1989. Recorrido y uso del espacio en un grupo de Lagothrix lagothricha (Primates: Cebidae) Mono Lanudo Churuco en la Amazonia colombiana. Trianea 3: 183-205.

Defler, T. R. 1991. Preliminary observations of Cacajao melanocephalus (Humboldt, 1811) (Primates, Cebidae). Trianea (Acta Cient. Tecn. INDERENA) 4:557-558.

Defler, T. R. 1996. Aspects of the ranging patterns in a group of wild woolly monkeys (Lagothrix 
lagotrhicha). American Journal of Primatology 38: 289-302.

Defler, T. R. 1999. Fision-fusion in the black-headed uacari (Cacajao melanocephalus) in Eastern Colombia. Neotropical Primates 7 (1): 5-8.

Defler, T. R. \& S. B. Defler. 1996. Diet of a group of Lagothrix lagotrhicha lagothricha in southeastern Colombia. International Journal of Primatology 17 (2): 161-190.

De Oliveira, A. A. \& B. W. Nelson. 2001. Floristic relationships of terra firme forests in the Brazilian Amazon. Forest Ecology and Management 36: 53-61.

Diazgranados, D. A. 1979. Geografía. En: Proyecto Radargramétrico del Amazonas (ed.). La Amazonia colombiana y sus recursos. Bogotá. Pp. 5-27.

Duivenvoorden, J. F. 1996. Patterns of tree species richness in rain forests of the middle Caqueta area, Colombia, NW Amazonia. Biotropica 28: 142-158.

Duque, Á., J. F. Phillips, P. Hildebrand, C. A. Posada, A. Prieto, A. Rudas, M. Suescún \& P. R. Stevenson. 2007. Distance decay of tree species similarity in protected areas on Terra Firme forests in Colombian Amazonia. 41: en prensa.

Fine, P. V. A., I. Mesones \& P. D. Coley. 2004. Herbivores promote habitat specialization by trees in amazonian forests. Science 305: 663-665.

Fisher, A. A., A. S. Corbet \& C. B. Williams. 1943. The relation between the number of species and the number of individuals in a random sample of an animal population. Journal of Animal Ecology 12: 42-58.

Gentry, A. H. 1988. Changes in Plant community diversity and floristic composition on environmental and geographical gradients. Annals of the Missouri Botanical Garden 75 (1): 1-34.

Gentry, A. H. 1992. Tropical forest biodiversity distributional patterns and their conservational significance. Oikos 63: 19-28.
González, M. 2006. Patrones de uso de hábitat, actividades y dieta de los micos churucos ( $\mathrm{La}$ gothrix lagothricha) en la Estación Biológica Caparú (Vaupés, Colombia). Tesis de pregrado. Departamento de Ciéncias Biológicas, Universidad de los Andes. Bogotá.

González, M. 2007. Los micos churucos (lagothrix lagothricha) como dispersores de semillas en la Estación Biológica Caparú (Vaupés, Colombia). Tesis de maestría. Departamento de Ciéncias Biológicas, Universidad de los Andes. Bogotá.

Ibarra, C., J. Morelo, J. Briceño, A. Cortés, B. Motta \& C. Pulido. 1979. Suelos. En: Proyecto Radargramétrico del Amazonas (ed.). La Amazonía colombiana y sus recursos. Bogotá. Pp. 93-216.

Jaramillo, C., M. J. Rueda \& G. Mora. 2006. Cenozoic Plant Diversity in the Neotropics. Science 311: 1893-1896.

Jones, M. M., H. Tuomisto, D. B. Clark \& P. Olivas. 2006. Effects of mesoscale environmental heterogeneity and dispersal limitation on floristic variation in rain forest ferns. Journal of Ecology 94: 157-170.

Krebs, C. J. 1999. Ecological Methodology. Addison Wesley Longman, Inc. California.

Lloyd, M. \& R. J. Ghelardi. 1964. A table for calculating the "equitability" component of species diversity. Journal of Animal Ecology 33: 217-225.

McCune, B. \& M. J. Mefford. 2006. PC-ORD. Multivariate analysis of ecological data. Version 5. мJM Software. Gleneden Beach, Oregon.

McIntosh, R. I. 1967. An index of diversity and the relation of certain concepts to diversity. Ecology 48: 392-404.

Mantilla-Meluk, H. \& L. F. Barrios-Rodríguez. 1999. Ecología básica de Cebus apella en la región del bajo Apaporis, Amazonia Colombiana. Tesis de Pregrado. Departamento de Biología, Facultad de Ciencias, Universidad Nacional de Colombia. Bogotá. 
Nebel, G., L. P. Kvist, J. K. Vanclay \& H. Vidaurre. 2001. Forest dynamic in flood plain forests in the Peruvian Amazon: effects of disturbance and implications for management. Forest Ecology and Management 150: 27-57.

Nebel, G., L. P. Kvist, J. K. Vanclay, H. Christensen, L. Freitas \& J. Ruiz. 2002. Structure and floristic composition of flood plain forests in the Peruvian Amazon I. Overstorey. Forest Ecology and Management 295: 666-669.

Palacios-Acevedo, E. \& A. Rodríguez-Rodríguez. 1995. Caracterización de la dieta y comportamiento alimentario de Callicebus torquatus lugens. Tesis de Pregrado. Departamento de Biología, Facultad de Ciencias, Universidad Nacional de Colombia. Bogotá.

Palacios, E., A. Rodríguez \& T. R. Defler. 1997. Diet of a group of Callicebus torquatus lugens (Humboldt, 1812) during the annual resource bottleneck in Amazonian Colombia. International Journal of Primatology 18 (4): 503-522.

Palacios, E. \& C. A. Peres. 2005. Primate population densities in three nutrient-poor Amazonian terra firme forest of south-eastern Colombia. Folia Primatologica 76 (3): 135-145.

Parolin, P., O. De Simone, K. Haase, D. Waldhoff, S. Rottenberger, U. Kuhn, J. Kesselmeier, B. Kleiss, W. Schmidt, M. T. F. Piedade \& W. J. Junk. 2004a. Central Amazonian Floodplain Forests: Tree Adaptations in a Pulsing System. The Botanical Review 70: 357-380.

Parolin, P., J. Adis, M. F. Da Silva, I. L. Do Amaral, L. Schmidt \& M. T. F. Piedade. 2004b. Amazonianan-Limnologia et oecologia regionalis systemae fluminis. Amazonas 85: 484-489.

Pitman, N. C. A., J. W. Terborgh, M. R. Silman, V. P. Nuñez, D. A. Neill, C. E. Cerón, W. A. Palacios \& M. Aulestia. 2002. A comparison of the tree species diversity in two upper amazonian forests. Ecology 83(11): 3210-3224.

Poulsen, A. D., H. Tuomisto \& H. Balslev. 2006. Edaphic and floristic variation within a 1-ha plot of lowland Amazonian rainforest. Biotropica 12: 1107-1138.
Rangel, E. \& B. Luengas. 1997. Clima y Aguas. En: IGAC (ed.). Zonificación ambiental para el plan modelo colombo-brasilero (eje Apaporis-Tabatinga: РАT). Editorial Linotipia Bolivar. Bogotá. Pp. 49-68.

SINCHI-Instituto Amazónico de Investigaciones Científicas. 2008. Sistema de Información Ambiental Territorial-Amazonía colombiana SIAT-AC. Disponible en: http://siac.net.co/ web/guest/region

Sørensen, T. 1948. A method of establishing groups of equal amplitude in plant society based on similarity of species content. K. Danske Vidensk. Selsk 5: 1-34.

Stevenson, P. R., M. Suescún \& M. J. Quiñones. 2004. Characterization of forest types at the CIEM, Tinigua Park, Colombia. Field studies of fauna and flora La Macarena, Colombia 14: 1-20.

Stropp, J., H. ter Steege, Y. Malhi, ATDN \& RAINFOR. 2009. Disentangling regional and local tree diversity in the Amazon. Ecography 32: 46-54.

Terborgh, J. \& E. Andresen. 1998. The composition of Amazonian forests: patterns at local and regional scale. Journal of Tropical Ecology 14: 645-664.

Ter Steege, H., D. Sabatier, H. Castellanos, T. van Andel, J. Duivenvoorden, A. A. De Oliveira, R. Ek, R. Lilwah, P. Maas \& S. Mori. 2000. An analysis of the floristic composition and diversity of Amazonian forests including those of the Guiana Shield. Journal of Tropical Ecology 16: 801-828.

Tuomisto, H., K. Ruokolainen, R. Kalliola, A. Linna, W. Danjoy \& Z. Rodríguez. 1995. Dissecting Amazonian biodiversity. Science 269: 63-66.

Vargas, I. N. 2008. Evaluación de aspectos de reclutamiento limitado y su efecto sobre la diversidad en un bosque húmedo tropical, Estación Biológica Caparú, Vaupés, Colombia. Tesis de maestría. Departamento de Ciencias Biológicas, Universidad de los Andes. Bogotá. 
Wills, C., R. Condit, R. B. Foster \& S. P. HubbeII. 1997. Strong density- and diversity-related effects help to maintain tree species diversity in a neotropical forest. Proceedings of the National Academy of Science of the United States of America 94: 1252-1257

Wittmann, F., J. Schöngart, J. C. Montero, T. Motzer, W. J. Junk, M. T. F. Piedade, H. L.
Queiroz \& M. Worbes. 2006. Tree species composition and diversity gradients in whitewater forests across the Amazon Basin. Journal of Biogeography 33: 1334-1347.

Wright, S. J. 2002. Plant diversity in tropical forests: a review of mechanisms of species coexistence. Oecologia 130: 1-14. 